\title{
Modelling and Dynamic Simulation of One-Dimensional Isothermal Axial Dispersion Tubular Reactors with Power Law and Langmuir-Hinshelwood- Hougen Watson Kinetics
}

\author{
Almoruf Olajide Fasola Williams* \\ Chemical and Petroleum Engineering Department, University of Laagos, Akoka, Lagos, \\ Nigeria
}

\begin{abstract}
In this paper, the modelling, numerical lumping and simulation of the dynamics of one-dimensional, isothermal axial dispersion tubular reactors for single, irreversible reactions with Power Law (PL) and Langmuir-Hinshelwood-Hougen-Watson (LHHW)-type kinetics are presented. For the PL-type kinetics, first-order and second-order reactions are considered, while Michaelis-Menten and ethylene hydrogenation or enzyme substrate-inhibited reactions are considered for the LHHW-type kinetics. The partial differential equations (PDEs) developed for the one-dimensional, isothermal axial dispersion tubular reactors with both the PL and LHHW-type kinetics are lumped to ordinary differential equations (ODEs) using the global orthogonal collocation technique. For the nominal design/operating parameters considered, using only 3 or 4 collocation points, are found to adequately simulate the dynamic response of the systems. On the other hand, simulations over a range of the design/operating parameters require between 5 to 7 collocations points for better results, especially as the Peclet number for mass transfer is increased from the nominal value to 100. The orthogonal collocation models are used to carry out parametric studies of the dynamic response behaviours of the one-dimensional, isothermal axial dispersion tubular reactors for the four reaction kinetics. For each of the four types of reaction kinetics considered, graphical plots are presented to show the effects of the inlet feed concentration, Peclet number for mass transfer and the Damköhler number on the reactor exit concentration dynamics to step-change in the inlet feed concentration. The internal dynamics of the linear (or linearized) systems are examined by computing the eigenvalues of the linear (or linearized) lumped orthogonal collocation models. The relatively small order of the lumped orthogonal collocation dynamic models make them attractive and useful for dynamic resilience analysis and control system analysis/design studies.
\end{abstract}

Keywords: Isothermal tubular reactors, power-law kinetics, Langmuir-Hinshelwood-Hougen-Watson kinetics, orthogonal collocation, dynamic simulation

\section{Introduction}

Reactors are at the heart of the chemical, oil and gas, fertilizer, petrochemical, biochemical and pharmaceutical industries. Various types are found depending on the application, mode of operation, number of phases involved and whether it is catalytic or non-catalytic [1]. Reactors can be classified into three types based on operational mode: batch reactors, semi-batch reactors and continuous

\footnotetext{
* Corresponding author. Tel.: +234-8059482712

E-mail address: afwilliams@unilag.edu.ng

Manuscript History: Received 24 April, 2021, Revised 12 July, 2021, Accepted 12 July, 2021, Published 31 October, 2021 Copyright (C) 2021 UNIMAS Publisher. This is an open access article under the CC BY-NC-SA 4.0 license.

https://doi.org/10.33736/jaspe.3328.2021
}

e-ISSN: 2289-7771 
reactors. The continuous reactors can be further sub-divided into continuously stirred tank reactors (CSTR), tubular reactors, fixed-bed reactors and fluidized-bed reactors, to mention a few [2-4].

Tubular reactors may be operated isothermally or non-isothermally. Isothermal reactor operations occur for negligible heat of reaction cases. For non-negligible heat of reaction, an isothermal operation may be achieved by placing the reactor tube in a bath or it could be a tube in a jacket configuration, or it could involve several tubes immersed in a heat transfer medium [5].

\subsection{Ideal and non-ideal tubular reactors}

The tubular plug flow reactor (TPFR) model is the ideal tubular reactor that is widely employed in many design and analysis tasks. In this reactor, the assumption is usually made that there is neither axial mixing nor radial gradients. However, in real industrial reactors, there is usually some deviation from this assumption as the flow may show some axial mixing and possibly radial gradients that will result in different system properties and hence lower the performance from what is obtainable with the ideal reactor. Factoring the variation of the system properties in the axial and radial directions into the model leads to a two-dimensional model which is more complicated to handle for both steady-state and dynamic or control studies especially during the early engineering design phase. Fortunately, it is generally the practice that when the length or diameter ratio of the tubular reactor is large, then one can neglect any system variation in the radial direction without any significant loss inaccuracy. In this case, the system effectively becomes one-dimensional with system variation considered only in the axial direction with the resulting simplicity in the subsequent simulation, analysis and control design. To account for the non-ideal behaviour of industrial tubular reactors, a single parameter known as the axial dispersion coefficient is introduced into the reactor model to characterize the collective action of all phenomena such as molecular diffusion, turbulent mixing, and non-uniform velocities, which leads to a distribution of residence times in the reactor [5]. The resulting model is then known as the onedimensional axial dispersion tubular reactor model $[2,6]$. As a result of its simplicity, this model is widely used in the literature describing the nonideal flow pattern in tubular reactors $[4,5]$.

The dynamics (or transient response behaviour) of the one-dimensional axial dispersion tubular reactors are modelled by partial differential equations (PDEs) of the parabolic-type, based on first principles or mechanistic modelling approach, and therefore belong to the class of systems known as distributed parameter systems (DPS). In these systems, the state/output and/or the input may vary both spatially and temporarily [7]. Knowledge of the system dynamics is important as it provides the basis for the analysis and design of control systems for the reactors during normal operation as well as during start-up and shut-down. It is of interest to be able to analyse such situations since operations during start-up and shut-down usually lead to the manufacture of products that do not meet the desired specifications [8].

Obtaining the system dynamics requires the solution of the PDEs modelling the system. Except for the simplest of cases where the PDE is linear or linearized around some steady-state profile in which an analytical solution is possible, the solution of the PDE must be solved numerically to determine the system dynamics. In doing this, an additional consideration is the desire to obtain suitable control-oriented models (i.e. low-order, accurate enough) which can be used for control system analysis and design. This approach is based on the early lumping method [7]. The attraction of this approach is the use of a simpler model that closely approximates the original DPS and consequently leads to a smaller number of equations to be solved. Additionally, one can then employ any of the large body of analysis or controller design methods for lumped parameter (LP) systems with much ease. The other approach, known as late lumping, involves the application of distributed parameter systems theory and the mathematical rigour required by this approach [7,9] is not attractive for industrial deployment for many practical processes. 


\subsection{Numerical methods for lumping of PDE models}

Many numerical methods based on lumping or model order reduction are available for obtaining the solution of PDEs which apply to linear and nonlinear models. Li and Qi [10] present a review of some of the key modelling and lumping techniques for distributed parameter systems, and cited several reported applications in the literature. For systems with known PDEs, the lumping/model reduction methods are categorized into the method of weighted residuals (MWR) such as the Galerkin method and the collocation method, and the approximate inertial manifold (AIM) technique. By combining these with the choice of the basis functions (e.g. finite difference methods, finite element methods, spectral methods and the Karhunen-Loeve method - also known as the proper orthogonal decomposition, POD method) different techniques arise. These techniques have different strengths and weaknesses depending on the problem at hand. For example, one of the popular methods that find wide application (because it generally works) is the classical finite difference technique in which both the time and spatial variables are discretized resulting in a system of algebraic equations that are then solved. In a variant of this technique known as the method of lines, only the spatial variables are discretized leading to a system of ordinary differential equations which can be integrated using a numerical integration routine to determine the system dynamic response. While these two techniques are known to be easy and general to apply, they are well known to be generally computationally inefficient, and often require high order system for accurate solution compared with other techniques such as the method of weighted residuals [10-13] of which the orthogonal collocation method is a popular one.

\subsection{Previous studies of dynamics of isothermal tubular reactors}

Apart from the related works cited by Li and Qi [10], a number of other studies of the dynamics and control of isothermal plug flow reactors and axial dispersion tubular reactors based on the application of the various model reduction methods highlighted above, have also appeared in the literature.

Inspired by the earlier work of Dochain et al. [14], Gomez et al. [15] presented the development of a finite-dimensional model for a class of nonlinear distributed parameter systems using the orthogonal collocation technique. The resulting model was feedback linearized and then used to develop an adaptive control algorithm to compensate for model mismatch from parametric and dynamic uncertainties of the reduced model. The approach was successfully applied in the simulation output tracking control of a fixed bed bioreactor operating isothermally.

Li [16] studied the dynamics of an isothermal axial dispersion tubular reactor with first-order kinetics based on the state-space approach using two methods - analytical and numerical. In the analytical approach, the PDE modelling the system is reduced to an approximate lumped parameter system using a modal decomposition method based on the system natural eigenfunctions. In the numerical approach, the PDE modelling the system is first discretized with respect to time using an implicit finite difference technique. The resulting high-order system is then reduced by using the proper orthogonal decomposition (POD) to extract the most important characteristic spatial profile. The resulting reduced-order models from the two approaches were then used as a basis for the control design to achieve an optimal trajectory of the feed concentration (with bounded constraints) in the reactor outlet concentration. The control problem was solved using quadratic programming. Alopaeus et al. [17] developed the dynamic model of a plug flow reactor (PFR) including multiple fluid and solid phases and used a variable-order, piece-wise moment conserving method to lump the PDE modelling the system to ODEs. Standard ODE integration routines were then used to obtain the state profile solutions. One of the examples considered was an isothermal PFR carrying out a second-order dimerization reaction. The method is particularly attractive for systems with sharp concentration gradients at the reactor inlet. 
Petre and Selisteanu [18] carried out the modelling, model reduction, simulation and nonlinear control design for a bioprocess (a fixed-bed bioreactor without axial dispersion) modelled by a system of PDEs. The model reduction was done using the orthogonal collocation method. They found that by appropriately choosing the collocation points along the fixed bed bioreactor, the resulting reducedorder model closely approximates the behaviour of the original PDE model. The controllers designed were based on input-output linearization and nonlinear adaptive control techniques. The performances of the controllers were tested via simulation of substrate concentration control for the fixed bed bioreactor and they reported excellent results. Rachidi et al. [19] also carried out the modelling and development of a reduced order model for a nonlinear fixed-bed bioreactor described by hyperbolictype PDEs using the orthogonal collocation method. The simulated dynamics of the system obtained by the orthogonal collocation method were compared with those obtained using the classical finite difference method. They reported that only four internal collocation points were sufficient to obtain acceptable simulation results compared with the finite difference solution that required 500 discretizations points.

Giwa and Giwa [20] simulated the dynamic response of an isothermal axial dispersion tubular reactor with first-order kinetics using the Crank-Nicholson finite difference method as well as explicit and implicit finite difference methods. They found, as expected, that the Crank-Nicholson method gave better results. More recently, Williams and Adeniyi [21] carried out the model reduction of a one-dimensional, isothermal axial dispersion tubular reactor with first-order kinetics using the modal decomposition method. It was found that a 3rd-order system was adequate to approximate the PDE system. The reduced-order system was then employed to design PID-type controllers for the system using a modified IMC-PID method [22]. The performance of the PID-type controllers was tested using closed-loop simulations with excellent results obtained. A shortcoming of the approach is that the modal decomposition method employed for the model reduction is only applicable to linear PDEs that are self-adjoint and with a wide spectrum of eigenvalues [7]. Conesa [23] presented the simulation of the dynamic behaviour of both the ideal plug flow tubular reactor and an axial dispersion, a one-dimensional tubular reactor with first-order reaction kinetics using the finite difference method with as many as 50 discretizations points for the former (i.e. ideal plug flow reactor) and 150 discretization points for the latter (i.e. for the axial dispersion tubular reactor).

Elhajaji et al. [24] presented a quadratic spline collocation method for the spatial lumping of one-dimensional nonlinear PDEs describing the dynamics of an ideal plug flow reactor. This was combined with discretization in time using the classical Crank-Nicholson technique. The method was applied to two examples, one of which was an ideal isothermal tubular reactor with irreversible sequential reaction in which the first reaction has second-order kinetics. In a related study, Barjes et al. [25] employed a cubic spline collocation for the same system investigated by Elhajaji et al. [24]. It is seen that the cubic spline collocation gave better accuracy than the quadratic spline collocation method, requiring from 5 to 10 spatial discretization points and between 10 and 20-time discretization points to give acceptable accuracy. A drawback of these two approaches is that they are only suited for simulations as they do not lead to models that can be employed for control system analysis and design i.e. the model reduction process do not lead to control-oriented models.

\subsection{Current study}

The foregoing cited works on isothermal tubular/fixed bed reactors show that, except for the few cited cases that employed the powerful method of weighted residual methods for dynamics and control studies, most of the other studies have generally used the finite difference method which is not attractive for simulations and control analysis and design. This is due to the resulting large size of the lumped parameter model when the finite difference method is employed to lump the PDE model into ODEs. This work can therefore be seen as a contribution to the existing body of knowledge about the dynamics of isothermal tubular reactors with the objectives of determining low-order lumped 
parameter models that can be useful for dynamic simulations, and control system analysis/design. This current study is focused on the modelling and dynamic simulation of a class of one-dimensional, isothermal axial dispersion tubular reactors carrying out single, irreversible reactions with power-law (PL) and Langmuir-Hinshelwood-Hougen-Watson (LHHW)-type kinetics. The PL- and LHHW-type kinetics describe many important reactions [4]. Two cases each of the PL-type kinetics (e.g. first- and second-order) and LHHW-type kinetics (Michaelis-Menten and Ethylene Hydrogenation or SubstrateInhibited kinetics) are considered. Thus, except for the system with first-order reaction kinetics which leads to a linear model, all the other three models are nonlinear and so can only be solved numerically. One of the objectives of the study is to obtain low order lumped parameter models of the systems considered which can be used for dynamic resilience analysis and control system analysis/design. Thus for this purpose, the orthogonal collocation method is adopted in lumping the PDE modelling the systems. The resulting ordinary differential equations (ODEs) are numerically integrated to determine the system dynamics to changes in the feed inlet and pertinent process parameters. Our choice of the orthogonal collocation method is motivated by the fact that it is well known to be an efficient and powerful reduction method that gives comparable accuracy with the Galerkin method; it is simple to implement, and there exist reliable computer routines to aid in its application [13]. Additionally, as noted by Dochain et al. [14, 26], Cho and Joseph [27] have shown that orthogonal collocation methods preserve mass balances. The second objective of this work is to carry out parametric studies of the transient response behaviours for a range of the characteristics parameters of the tubular reactor models. This shall be done using the lumped parameter models obtained from the application of the orthogonal collocation method. This kind of simulation can provide important information and insights about the behaviours of the systems under different design and operating conditions which result in better design, operation or control.

\section{Materials and methods}

\subsection{Model of isothermal tubular reactor systems with axial mass dispersion}

Consider the homogeneous (or pseudo-homogeneous), one-dimensional tubular reactor system is shown in schematically in Figure 1, in which the irreversible chemical reaction $\mathrm{A} \rightarrow \mathrm{B}$ is taking place.

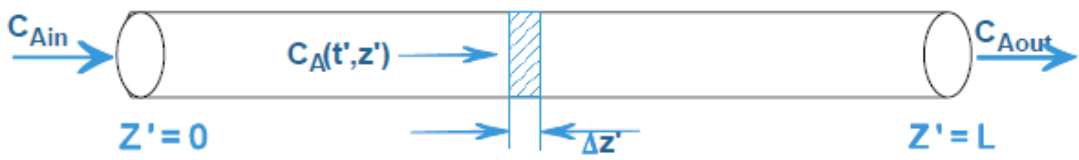

Figure 1: Schematic of a tubular reactor system

By making the following assumptions: (i) isothermal operation, (ii) uniform cross-sectional area, (iii) constant fluid properties, (iv) constant dispersion coefficient $\mathrm{D}$, and that (v) radial gradients in velocity and concentration are negligible; and writing a microscopic material balance for component A, one obtains the mathematical model $[4,5]$ :

$$
\frac{\partial c_{A}}{\partial t^{\prime}}=D \frac{\partial^{2} c_{A}}{\partial z^{\prime 2}}-v \frac{\partial c_{A}}{\partial z^{\prime}}-r_{A}
$$

where $D$ is the axial mass dispersion coefficient, an empirical quantity. 
Butt [8] has discussed and summarized the considerable body of experimental information concerning values of D for various types of flow situations, which include: laminar and turbulent flow of both gases and liquids in empty tubes, fixed and fluidized beds, and extraction columns.

Four different types of boundary conditions can be written [8] for use with Eq. (1). However, using different mathematical arguments, Butt [8] and Villadsen and Michelsen [13] have shown that the only correct boundary conditions are those proposed by Danckwerts [28], given by the following:

$$
\begin{gathered}
\text { At } z^{\prime}=0, \quad v\left(c_{A}-c_{A i n}\right)=D\left(\frac{\partial c_{A}}{\partial z^{\prime}}\right)_{z^{\prime}=0} \\
\text { At } \quad z^{\prime}=L, \quad\left(\frac{\partial c_{A}}{\partial z^{\prime}}\right)_{z^{\prime}=0}=0
\end{gathered}
$$

The use of these boundary conditions for Eq. (1) yields a solution that reduces to that of the tubular plug flow reactor model (TPFRM) in the limit of $\mathrm{D} \rightarrow 0$, and to that of the continuous stirred tank reactor (CSTR) in the limit $\mathrm{D} \rightarrow \infty$ [8].

An appropriate initial condition for Eq. (1) is:

$$
\text { At } t^{\prime}=0, \quad c_{A}\left(z^{\prime}, 0\right)=\bar{c}_{A}\left(z^{\prime}\right)
$$

Having derived this general mathematical model of the isothermal tubular reactor with axial mass dispersion, the applicable models for two classes/types of rate equations (i.e. PL and LHHW kinetics) for the irreversible reaction under consideration will now be presented.

Although the foregoing model is for an empty tubular reactor, the pseudo-homogeneous model case implies that the same model can be applied to a packed-bed reactor where there are no fluid-tocatalyst particle mass and/or heat transfer resistances or they are considered small. Pseudohomogeneous tubular reactor models are widely employed in the literature to simulate, design/optimize and control catalytic fixed bed reactors [29-40]. This is because pseudohomogeneous models are much simpler to use for simulation, optimization or control design since the inter-and intra-particle resistances are neglected. Such simplifications are known to give comparable results with the full heterogeneous models for isothermal operations or when the heat effects for a non-isothermal reactor is not severe $[32,33,35,41]$. This is attractive since we get a much simpler model that can be employed for optimization and control analysis/design studies prior to the detailed design phase where more accurate models may be utilized.

\subsubsection{Irreversible Nth. order reaction i.e. power-law kinetics}

Power law kinetics are frequently used to quantify homogeneous reactions [42]. For irreversible Nth. order reaction, the rate equation takes the form:

$$
r_{A}=k c_{A}^{N}
$$

which when substituted into Eq. (1) gives:

$$
\frac{\partial c_{A}}{\partial t^{\prime}}=D \frac{\partial^{2} c_{A}}{\partial z^{\prime 2}}-v \frac{\partial c_{A}}{\partial z^{\prime}}-k c_{A}^{N}
$$


If we define the following variables and parameters:

$$
\begin{gathered}
z=z^{\prime} / L, \quad t=\frac{v}{L} t^{\prime}, \quad x=\frac{c_{A}}{c_{r e f}} \\
D a=\frac{L k c_{r e f}^{N-1}}{v} \text { and } P e_{m}=\frac{L v}{D}
\end{gathered}
$$

then, Eq. (6) becomes:

$$
\frac{\partial x}{\partial t}=\frac{1}{P e_{m}} \frac{\partial^{2} x}{\partial z^{2}}-\frac{\partial x}{\partial z}-D_{a x}{ }^{N}
$$

and the corresponding initial and boundary conditions become

$$
\begin{gathered}
\text { At } t=0, \quad x(z, 0)=x_{0}(z) \\
\text { At } z=0, \quad x-\frac{1}{P e_{m}} \frac{\partial x}{\partial z}=x_{i n}(t) \\
\text { At } \quad z=1, \quad \frac{\partial x}{\partial z}=0
\end{gathered}
$$

The parameter, $\mathrm{Da}$, known as the Damköhler number, is a measure of the rate of reaction at inlet conditions; while the parameter, Pem, commonly used to represent the magnitude of the dispersion effects, is known as the axial Peclet number for mass transfer. The Peclet number, Pe, is the ratio of characteristic constants for convective and dispersive effects.

For the special case of a first-order reaction:

Then Eq. becomes

$$
N=1 \quad \text { and } \quad D a=\frac{L k}{v}
$$

$$
\frac{\partial x}{\partial t}=\frac{1}{P e_{m}} \frac{\partial^{2} x}{\partial z^{2}}-\frac{\partial x}{\partial z}-D a x
$$

which is a second-order, linear, parabolic partial differential equation, subject to the same initial and boundary conditions given by Eqs. (9) to (10).

Fogler [4] gives an example of an industrially important first-order reaction as the cracking of ethane to ethylene in an isothermal tubular reactor at $1100 \mathrm{~K}$ according to the reaction:

$\mathrm{C}_{2} \mathrm{H}_{6} \rightarrow \mathrm{C}_{2} \mathrm{H}_{4}+\mathrm{H}_{2}$.

For the second-order reaction:

$$
N=2 \quad \text { and } \quad D a=\frac{L k}{v}
$$


Then Eq. becomes

$$
\frac{\partial x}{\partial t}=\frac{1}{P e_{m}} \frac{\partial^{2} x}{\partial z^{2}}-\frac{\partial x}{\partial z}-D_{a x}^{2}
$$

which is a second-order, nonlinear, parabolic partial differential equation, subject to the same initial and boundary conditions given by Eqs. (8) to (10).

Fogler [4] gives the following examples of second-order reactions: (a) the dimerization reaction $2 \mathrm{~A} \rightarrow \mathrm{B}$, (b) the isomerization reaction $\mathrm{A} \rightarrow \mathrm{B}$ and (c) the gas-phase reaction $2 \mathrm{CH}_{3} \mathrm{OH} \rightarrow \mathrm{C}_{2} \mathrm{H}_{4}+$ $2 \mathrm{H}_{2} \mathrm{O}$ over a zeolite catalyst for the production of olefins from methanol.

\subsubsection{Reactions with Langmuir-Hinshelwood-Hougen-Watson type kinetics}

For irreversible reactions, $\mathrm{A} \rightarrow \mathrm{B}$ which follow the Langmuir-Hinshelwood-Hougen-Watson (LHHW) type kinetics, Carberry [43], gives a general rate expression as:

$$
r_{A}=\frac{k c_{A}^{M}}{\left(1+K c_{A}\right)^{N}}
$$

This type of expression is known to describe the reaction kinetics of many industrially important solid-catalysed reactions such as ethylene hydrogenation, and enzyme catalysed reactions such as fermentation of sucrose into simple sugars, and substrate-inhibited enzyme reactions. Note that the first- and second-order kinetics cases (i.e. power law kinetics type) previously presented can be considered as special cases of the LHHW type kinetics in which $\mathrm{M}=1, \mathrm{~N}=0$ and $\mathrm{M}=2, \mathrm{~N}=0$, respectively.

Substituting Eq. (13) into Eq. (1) gives:

$$
\frac{\partial c_{A}}{\partial t^{\prime}}=D \frac{\partial^{2} c_{A}}{\partial z^{\prime 2}}-v \frac{\partial c_{A}}{\partial z^{\prime}}-\frac{k c_{A}^{M}}{\left(1+K c_{A}\right)^{N}}
$$

subject to the same initial and boundary conditions given by Eqs. (2) and (4).

If we define similar parameters and variables as follows:

$$
\begin{gathered}
z=z^{\prime} / L, \quad t=\frac{v}{L} t^{\prime}, \quad x=K c_{A} \\
P e_{m}=\frac{L v}{D} \quad \text { and } \quad D a^{\prime}=\frac{L k K}{v K^{M}}=\frac{L k}{v K^{M-1}}
\end{gathered}
$$

then Eq. can be transformed into

$$
\frac{\partial x}{\partial t}=\frac{1}{P e_{m}} \frac{\partial^{2} x}{\partial z^{2}}-\frac{\partial x}{\partial z}-\frac{D a^{\prime} x^{M}}{(1+x)^{N}}
$$

Two practical examples of reactions with LHHW type kinetics are now considered; (1) reactions with Michaelis-Menten type kinetics and (2) ethylene hydrogenation reaction or enzyme-catalysed reaction with substrate-inhibition kinetics. For reactions with MichaelisMenten type kinetics, the rate expression for many enzyme-catalysed reactions such as fermentation of sucrose into simple sugars [2] or solid-catalysed reactions such as decomposition of ammonia on platinum, and of methane on carbon [44] is: 


$$
r_{A}=\frac{k_{1} c_{A}}{k_{2}+c_{A}}
$$

which is a special case of Eq. (13) with $M=1, N=1, k=k_{1} / k_{2}$ and $K=1 / k_{2}$. For this case, Eq. (15) reduces to

$$
\frac{\partial x}{\partial t}=\frac{1}{P e_{m}} \frac{\partial^{2} x}{\partial z^{2}}-\frac{\partial x}{\partial z}-\frac{D a^{\prime} x}{(1+x)}
$$

where $\mathrm{Da}^{\prime}=\mathrm{Da}=L k / v$.

For the ethylene hydrogenation reaction or enzyme-catalysed reaction with substrate-inhibition kinetics, the kinetic rate equation for ethylene hydrogenation in the presence of excess hydrogen [45], carbon monoxide oxidation on a platinum catalyst [12], or enzyme-catalysed reaction with substrate inhibition [46], is given by an equation of the form:

$$
r_{A}=\frac{k c_{A}}{\left(1+K c_{A}\right)^{2}}
$$

which is also a special case of Eq. (13) with $M=1, N=2$.

For this case, Eq. (15) reduces to:

where $\mathrm{Da}^{\prime}=\mathrm{Da}=L k / v$.

$$
\frac{\partial x}{\partial t}=\frac{1}{P e_{m}} \frac{\partial^{2} x}{\partial z^{2}}-\frac{\partial x}{\partial z}-\frac{D a^{\prime} x}{(1+x)^{2}}
$$

Eqs. (17) and (19) are also second-order, nonlinear, parabolic partial differential equations, subject to the same initial and boundary conditions given by Eqs. (8) to (10).

\subsection{Numerical lumping of the isothermal axial dispersion tubular reactor models using orthogonal collocation}

In this section, the numerical lumping of the various reactor models presented in Section 2.1 will be carried out. For the nonlinear models, orthogonal collocation shall be used to lump the nonlinear PDE models to ODEs which shall then be used for subsequent analysis and dynamic simulations. For the linear problem (i.e. isothermal tubular reactor with first-order reaction kinetics), analytical solutions of the steady-state and dynamic models are possible. For the linear dynamic model, the transient response solution using the modal decomposition technique is readily obtainable. Thus, the accuracy of the results of the orthogonal collocation solution for the linear problem shall be assessed using these analytical solutions.

The orthogonal collocation method is an attractive technique for obtaining an approximate solution of differential equations by fitting a trial solution at selected points. It belongs to the method of weighted residuals [11-13]. The method attempts to minimize the residuals that result when the trial solution is substituted into the differential equations modelling the system, and the residuals are set to zero at selected points (known as the collocation points) in the spatial direction of interest. By choosing the collocation points to be zeros of some orthogonal polynomials, the accuracy of the solution is greatly improved [13]. The details of the orthogonal collocation method can be found in Finlayson [11, 12], Villadsen and Michelsen [13]. A recent overview is presented by Young [47]. 


\subsubsection{Reactors with first-order kinetics} form

To solve Eq. (11) numerically using orthogonal collocation, we assume a trial solution of the

$$
x(z, t)=\sum_{k=0}^{n+1} \ell_{k}(z) x_{k}(t)
$$

in which $\ell_{k}(z), k=0,1,2, \ldots, n+1$ are Lagrange polynomials[13].

Substituting this into Eq. (11) and setting the residuals to zero at the collocation points leads to

$$
\frac{d x_{j}}{d t}=\frac{1}{P e_{m}} \sum_{k=0}^{n+1} B_{j k} x_{k}-\sum_{k=0}^{n+1} A_{j k} x_{k}-\operatorname{Dax}_{j}, j=1,2, \ldots, n
$$

in which $A_{j k}$ and $B_{j k}$ are the first and second collocation matrices [13], defined as:

$$
\begin{gathered}
A_{j k}=\left.\frac{d \ell_{k}(z)}{d z}\right|_{z=z_{j}} \\
B_{j k}=\left.\frac{d^{2} \ell_{k}(z)}{d z^{2}}\right|_{z=z_{j}}
\end{gathered}
$$

and the collocation points $z_{j}, j=0,1, \ldots, n-1$ are the roots of the orthogonal Jacobi Polynomial [13]:

$$
\int_{0}^{1} z^{\beta}(1-z)^{\alpha} z^{j} P_{n}^{(\alpha, \beta)}(z) d z=0, \quad \alpha, \beta>-1, j=0,1, \ldots, n-1
$$

Applying the trial solution to the boundary equations, Eqs. (9) and (10) become:

$$
\begin{gathered}
\sum_{k=0}^{n+1} A_{0, k} x_{k}=P e_{m}\left(x_{0}-u\right) \\
\sum_{k=0}^{n+1} A_{n+1, k} x_{k}=0
\end{gathered}
$$

Solving for $x_{0}$ and $x_{n+1}$ from Eqs. (25) and (26), and using them to eliminate $x_{0}$ and $x_{n+1}$ from Eq. (21), we have the following:

$$
\begin{array}{r}
x_{0}=-\left[L_{11} P e_{m} u+\left(L_{11} \sum_{k=1}^{n} A_{0, k} x_{k}+L_{12} \sum_{k=1}^{n} A_{n+1, k} x_{k}\right)\right] \\
x_{n+1}=-\left[L_{21} P e_{m} u+\left(L_{21} \sum_{k=1}^{n} A_{0, k} x_{k}+L_{22} \sum_{k=1}^{n} A_{n+1, k} x_{k}\right)\right] \\
\frac{d x_{j}}{d t}=\sum_{k=1}^{n}\left[\frac{1}{P e_{m}} B_{j k}-A_{j k}-D a \delta_{j k}+\left[\left(A_{j, 0}-\frac{B_{j, 0}}{P e_{m}}\right) L_{11}+\left(A_{j, n+1}-\frac{B_{j, n+1}}{P e_{m}}\right)\right] L_{21}\right] A_{0, k}
\end{array}
$$




$$
\begin{aligned}
& \left.+\left[\left(A_{j, 0}-\frac{B_{j, 0}}{P e_{m}}\right) L_{12}+\left(A_{j, n+1}-\frac{B_{j, n+1}}{P e_{m}}\right) L_{22}\right] A_{n+1, k}\right] x_{k} \\
& +\left[\left(P e_{m} A_{j, 0}-B_{j, 0}\right) L_{11}+\left(P e_{m} A_{j, n+1}-B_{j, n+1}\right) L_{21}\right] u
\end{aligned}
$$

in which:

$$
\begin{gathered}
\delta_{j k}= \begin{cases}0 & j \neq k \\
1 & j=k\end{cases} \\
L=\left[\begin{array}{ll}
L_{11} & L_{12} \\
L_{21} & L_{22}
\end{array}\right]=\left[\begin{array}{cc}
A_{0,0}-P e_{m} & A_{0, n+1} \\
A_{n+1,0} & A_{n+1, n+1}
\end{array}\right]^{-1}
\end{gathered}
$$

Putting Eq. (29) in the standard state-space form, we have

$$
\begin{gathered}
\frac{d \mathbf{x}}{d t}=\mathbf{A}_{1} \mathbf{x}+\mathbf{b}_{1} u \\
y=x_{n+1}=\mathbf{C x}+E_{1} u
\end{gathered}
$$

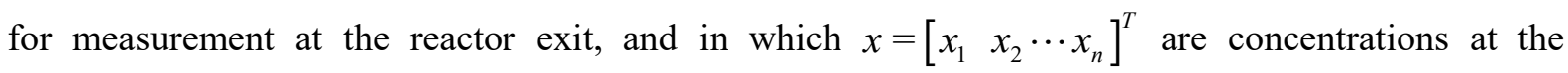
collocation points,

$$
\begin{gathered}
\mathbf{A}_{1}=\left[a_{1_{j k}}\right], j, k=1,2, \ldots, n \\
a_{1_{j k}}=\left[\frac{1}{P e_{m}} B_{j k}-A_{j k}-D a \delta_{j k}+\left[\left(A_{j, 0}-\frac{B_{j, 0}}{P e_{m}}\right) L_{11}+\left(A_{j, n+1}-\frac{B_{j, n+1}}{P e_{m}}\right) L_{21}\right] A_{0, k}\right. \\
\left.+\left[\left(A_{j, 0}-\frac{B_{j, 0}}{P e_{m}}\right) L_{12}+\left(A_{j, n+1}-\frac{B_{j, n+1}}{P e_{m}}\right) L_{22}\right] A_{n+1, k}\right] \\
\mathbf{b}_{1}=\left[b_{1_{1}} b_{1_{2}} \cdots b_{1_{n}}\right]^{T}=\left[\left(P e_{m} A_{j, 0}-B_{j, 0}\right) L_{11}+\left(P e_{m} A_{j, n+1}-B_{j, n+1}\right) L_{21}\right], \quad j=1,2, \ldots, n
\end{gathered}
$$

The steady-state solution can be obtained by setting the left-hand side of Eq. (31) to zero and solving for $\bar{x}$ which results in

$$
\overline{\mathbf{x}}=-\mathbf{A}_{1}^{-1} \mathbf{b}_{1} \bar{u}
$$

Since this system is linear, it is possible to derive an analytical solution for the steady-state and dynamic response behaviours of the system which can be used to make comparisons with the orthogonal collocation-based numerical solution. The steady-state and unsteady-state analytical solutions presented in Williams and Adeniyi [21] are used for this purpose. 


\subsection{Reactors with nonlinear reaction kinetics}

Unless the reactor model is linearized about a steady-state profile, $x^{-}$, in each of these cases (i.e. second-order, Michaelis-Menten and Ethylene-hydrogenation or substrate-inhibited kinetics), it is not possible to obtain an analytical solution to the corresponding reactor models. However, our objective is to solve the nonlinear problem rather than a linearized problem and it is only possible to do this numerically. The orthogonal collocation technique is applied to do this as follows.

For brevity, we shall consider an application to the axial dispersion reactor with the LHHW kinetics and derive the requisite equations for the second-order and Michaelis-Menten kinetics as special cases of this.

To solve this system using the orthogonal collocation technique, we assume a trial solution of the form of Eq. (20) and following the same procedure as for the first-order kinetics, the nonlinear PDE model, Eq. (15) is lumped to the nonlinear set of ODEs:

$$
\begin{aligned}
& \frac{d x_{j}}{d t}=F_{j}=\sum_{k=1}^{n}\left[\frac{1}{P e_{m}} B_{j k}-A_{j k}+\left[\left(A_{j, 0}-\frac{B_{j, 0}}{P e_{m}}\right) L_{11}+\left(A_{j, n+1}-\frac{B_{j, n+1}}{P e_{m}}\right) L_{21}\right] A_{0, k}\right. \\
& \left.+\left[\left(A_{j, 0}-\frac{B_{j, 0}}{P e_{m}}\right) L_{12}+\left(A_{j, n+1}-\frac{B_{j, n+1}}{P e_{m}}\right) L_{22}\right] A_{n+1, k}\right] x_{k}-D a^{\prime} \frac{x_{j}^{M}}{\left(1+x_{j}\right)^{N}} \\
& +\left[\left(P e_{m} A_{j, 0}-B_{j, 0}\right) L_{11}+\left(P e_{m} A_{j, n+1}-B_{j, n+1}\right) L_{21}\right] u \\
& j=1,2, \ldots, n
\end{aligned}
$$

At steady-state, the following nonlinear algebraic equations have to be solved for $\bar{x}_{j}$ :

$$
\begin{aligned}
& \sum_{k=1}^{n}\left[\frac{1}{P e_{m}} B_{j k}-A_{j k}+\left[\left(A_{j, 0}-\frac{B_{j, 0}}{P e_{m}}\right) L_{11}+\left(A_{j, n+1}-\frac{B_{j, n+1}}{P e_{m}}\right) L_{21}\right] A_{0, k}\right. \\
& \left.+\left[\left(A_{j, 0}-\frac{B_{j, 0}}{P e_{m}}\right) L_{12}+\left(A_{j, n+1}-\frac{B_{j, n+1}}{P e_{m}}\right) L_{22}\right] A_{n+1, k}\right] \bar{x}_{k}-\frac{D a \bar{x}_{j}^{M}}{\left(1+\bar{x}_{j}\right)^{N}} \\
& +\left[\left(P e_{m} A_{j, 0}-B_{j, 0}\right) L_{11}+\left(P e_{m} A_{j, n+1}-B_{j, n+1}\right) L_{21}\right] \bar{u}=0, \\
& j=1,2, \ldots, n
\end{aligned}
$$

The applicable equations for the three axial dispersion tubular reactors with nonlinear kinetics can be obtained from the foregoing equations by setting and substituting for the values of $\mathrm{M}$ and $\mathrm{N}$ as follows:

- Second-order kinetics: $M=2, N=0$

- Michaelis-Menten kinetics: $M=1, N=1$

- Ethylene Hydrogenation or Substrate-inhibited kinetics, $M=1, N=2$ 


\subsection{Method of solution for the linear and nonlinear orthogonal collocation models}

\subsubsection{Linear system}

The steady-state solution of the linear system given by Eq. (35) is solved using the Gaussian elimination routine of Villadsen and Michelsen [13]. If the problem has been properly formulated, $\mathbf{A}_{1}^{-1}$ exists and the Gaussian elimination algorithm determines the steady-state solution xs at the collocation points. This is known to be computationally more efficient than using the matrix inverse approach. The steady-state solutions at the collocation points serve as the initial conditions for the dynamic response simulations.

The dynamic response behaviour of the linear system is given by Eqs. (31 and 32) is obtained by carrying out discrete-time simulation using the state-transition method. This is also known to be computationally more efficient than carrying out numerical integration [48, 49]. The process is summarized as follows:

The continuous-time system given by Eq. (31) is converted to the discrete-time equivalent given by:

$$
\begin{gathered}
\mathbf{x}(k+1)=\mathbf{F} x(k)+\mathbf{G} u(k) \\
y(k+1)=\mathbf{C} \mathbf{x}(k+1)
\end{gathered}
$$

where $F$ and $G$ are computed using the basic Taylor series expansion method [50]:

$$
\begin{gathered}
\mathbf{F}=e^{\mathbf{A}_{1} \tau}=\sum_{k=0}^{\infty} \frac{1}{k !}\left(\mathbf{A}_{1} \tau\right)^{k} \\
\mathbf{G}=\int_{0}^{\tau} e^{\mathbf{A}_{1} t} \mathbf{b}_{1} d t=\tau\left[\sum_{k=0}^{\infty} \frac{1}{(k+1) !}\left(\mathbf{A}_{1} \tau\right)^{k}\right] \mathbf{b}_{1}
\end{gathered}
$$

Williams and Adeniyi [51] describe FORTRAN 77 routines developed to carry out the computation of the exponential matrix and its integral reliably and accurately.

\subsubsection{Nonlinear systems}

The set of nonlinear algebraic equations (Eq. 37) for the steady-state solutions (i.e. $\left.\bar{x}_{j}, j=1,2, \ldots, n\right)$ at the collocation points are solved by using the standard multivariable NewtonRaphson algorithm [52] for each of the cases: second-order, Michael-Menten and Ethylene or Substrate-inhibited kinetics. The steady-state analytical solution of the linear system model [21] evaluated at the collocation points, or the steady-state collocation (numerical) solution of the linear problem may be used as initial guess values for the Newton-Raphson algorithm. Both methods give equivalent results. However, using the steady-state analytical solution (at the collocation points) as the initial guess values for the Newton-Raphson algorithm, especially for simulation for a range of system design and operating parameters (i.e. parametric studies as presented later under the Results and Discussion section of this paper) cuts down on the overall computing time, then when the steady-state collocation (numerical) solution of the linear problem is used. This is expected because using the latter method requires the solution of a set of linear algebraic equations, while the former method involves only simple function evaluations. 
Using the initial steady-state solution, $\bar{x}_{j}, j=1,2, \ldots, n$; the set of nonlinear ODEs given by Eq. (36) are integrated numerically using the 3rd-order semi-implicit Runge-Kutta method routines [13] to determine the transient response of $x_{j}(t)$ to the input, $u(t)$. When required, the eigenvalues of the linear (or linearized) lumped orthogonal models are computed using the QR routine [13].

\section{Results and discussions}

\subsection{Dynamic Response Simulation of Nominal Systems}

The dynamic response simulations of the isothermal axial dispersion tubular reactors with the various kinetics (i.e. first-order, second-order and LHHW-type kinetics) are presented in this section for the nominal system parameters of an isothermal tubular reactor system studied by [53]: Pem $=1.0$ and $\mathrm{Da}=2.0$.

Based on the recommendations of Georgakis et al. [54] and Villadsen and Michelsen [13] for parabolic partial differential equations, we chose $\alpha=0, \beta=0$ in all the orthogonal collocation solutions. Where necessary, computation of the system steady-state and dynamic response behaviour at fixed grid points other than the collocation points were obtained by Lagrangian interpolation.

For a step-change in the inlet reactor concentration from $\bar{u}=1.0$ to 3.0, Figures 2A-D show the simulated results of the dynamic response of the reactor exit concentration for first-order, secondorder, Michaelis-Menten and ethylene hydrogenation kinetics, respectively. In the case of Figure 2A, a comparison of the exact response obtained by modal decomposition [21] is made against the 4-point collocation solution, while in Figures 2B-D, comparisons of the 4-point and 8-point collocation solutions are shown. We can observe that these responses are smooth and do not display any unusual behaviour. Although not shown, the comparison plots of the 3-point collocation solutions also show a good approximation to the higher-order solutions. 

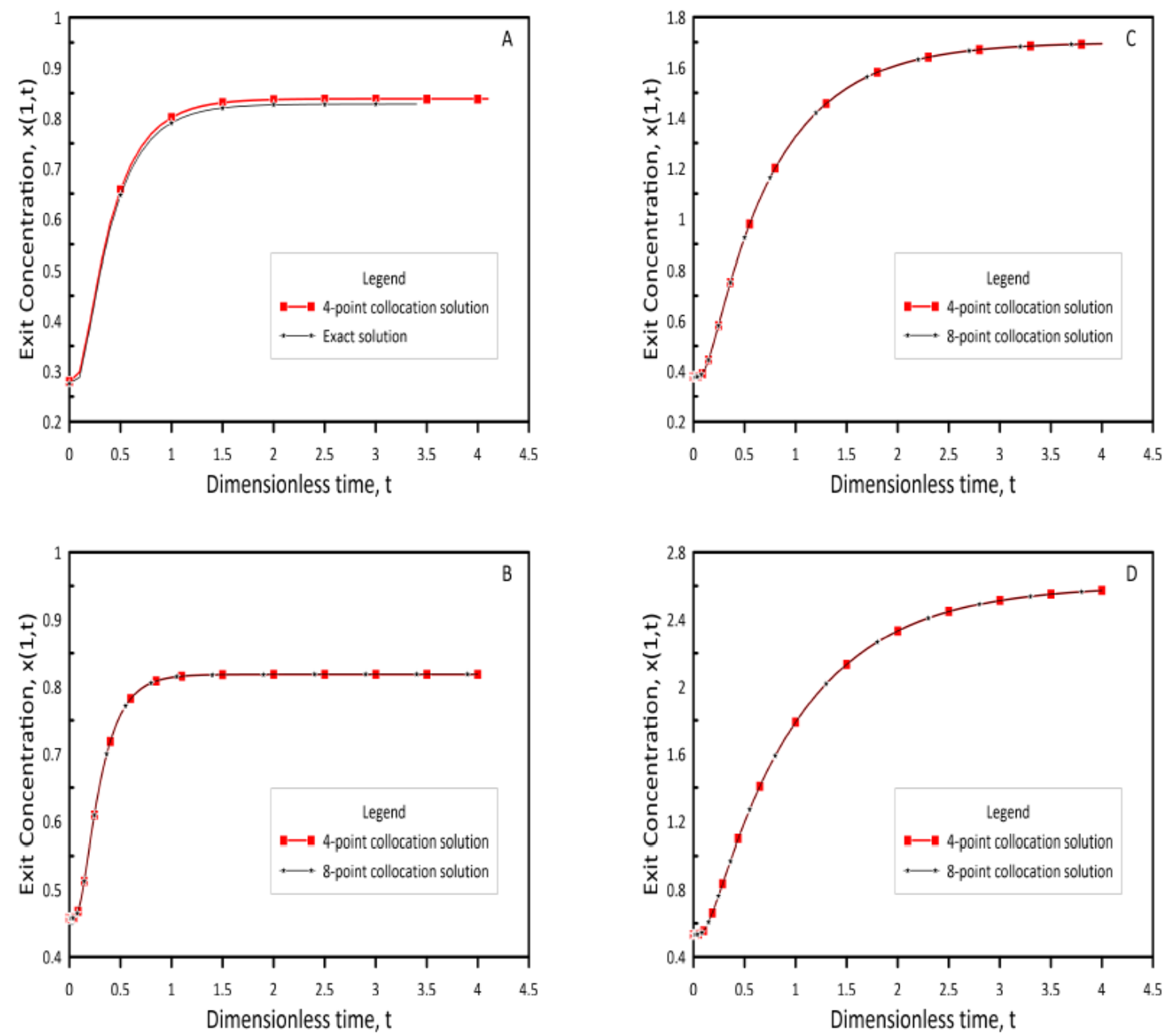

Figure 2: Response of exit reactor concentration to a step change of inlet concentration from $\bar{u}=1.0$ to 3.0 using orthogonal collocation. A: First-order; B: Second-order; C: Michaelis-Menten and D:

Ethylene hydrogenation kinetics. $\mathrm{Pem}=1.0, \mathrm{Da}=2.0$

\subsection{Parametric studies}

The nominal parameter values for the calculations whose results are presented below were as follows: $\bar{u}=1.0, \mathrm{Pem}=1.0$ and $\mathrm{Da}=2$. For each investigation, two of these parameters are kept at their nominal values and the third is varied over the desired range.

The parametric studies is carried out to determine the dynamic response behaviours of the reactors (with different kinetics) to variation of the steady-state inlet concentration $(\bar{u}=0.5,1.0,5.0,10.0)$, Peclet number for mass transfer $(\mathrm{Pem}=0.5,1.0,10.0,100.00)$, and Damköhler number $(\mathrm{Da}=0.5,1.0,5.0,10)$. For all these simulations, 7 internal collocation points were used because preliminary simulations indicated that the numerical dynamic solutions were better for increasing values of Pem. For each of these cases, the eigenvalues of the linear (or linearized) lumped orthogonal collocation models are computed to gain some insights into the internal dynamics of the systems for the four types of kinetics under consideration. 


\subsubsection{Effect of steady-state control input, $\bar{u}$}

An examination of the set of eigenvalues (of the linear or linearized models) given in Table 1 shows that they are all negative, implying the reactor is open-loop stable for all the four types of reaction kinetics operating at the steady-states defined by $\bar{u}$ and the nominal Pem and $\mathrm{Da}$. It can also be observed that at the nominal and other values of Pem and $\mathrm{Da}$, all the computed eigenvalues are real. This implies a diffusion- or dispersive controlled process.

As expected, the eigenvalues are independent of the steady-state input $(\bar{u})$ for the reaction with first-order kinetics, but these vary for the other three cases of nonlinear reaction kinetics. In all cases, it is clear that the system transient response will be dominated by the last three or four eigenvalues with the largest magnitudes. This is because the contribution of the other relatively large but negative eigenvalues to the system transient response will quickly die out. Thus, it is sufficient to look at the effect of $\mathrm{u}^{-}$on only the dominant eigenvalues.

For the reaction with second-order reaction kinetics, the effect of increasing $\bar{u}$ appears to significantly make the dominant eigenvalues more negative, implying a faster transient response. However, the reverse is the case for the reactor with Michaelis-Menten and ethylene hydrogenation reaction kinetics. For these, it can be seen that increasing $\bar{u}$ makes the dominant eigenvalues more positive, implying a slower transient response. It is observed that in these two cases, the changes in the dominant eigenvalues are not as pronounced as in the case of the first- and second-order reaction kinetics. Hence, one may not obtain any significant changes in the system transient response for these systems as $\bar{u}$ is varied.

Figure 3 shows the simulated transient response of the reactant exit concentration, $x(1, t)$ to a step change of +2 units in the inlet control input $(\mathrm{u})$ when the reactor is operating at different initial steady states defined by $\bar{u}$ and the nominal Pem and Da values. As expected, we can immediately see that the system transient step response for the case of first-order reaction kinetics is independent of $\bar{u}$ as shown in Figure 3A. Although settling at different steady-states defined by $\bar{u}$, the transient response shapes are all similar.

As $\bar{u}$ is increased for the second-order reaction kinetics, the system transient response becomes faster, while for the Michaelis-Menten and ethylene hydrogenation kinetics, the effect of increasing $\bar{u}$ though not appreciable, appears to be to slow the system dynamic response as depicted by the slow rise of the transient response to the final steady-states.

\subsubsection{Effect of Peclet number for mass transfer, Pem}

An examination of the set of eigenvalues (of the linear or linearized models) given in Table 2 shows they are all negative. This implies that the reactor is open-loop stable for the four types of reaction kinetics operating at the steady-states defined by the nominal $\bar{u}$, Da and range of Pem considered. It can be observed that the eigenvalues for the four types of reaction kinetics are of comparable values. This is expected because, at the low steady-state inlet concentration defined by $\bar{u}$ $=1.0$, all the four types of reaction kinetics can effectively be approximated as first-order reaction kinetics. 

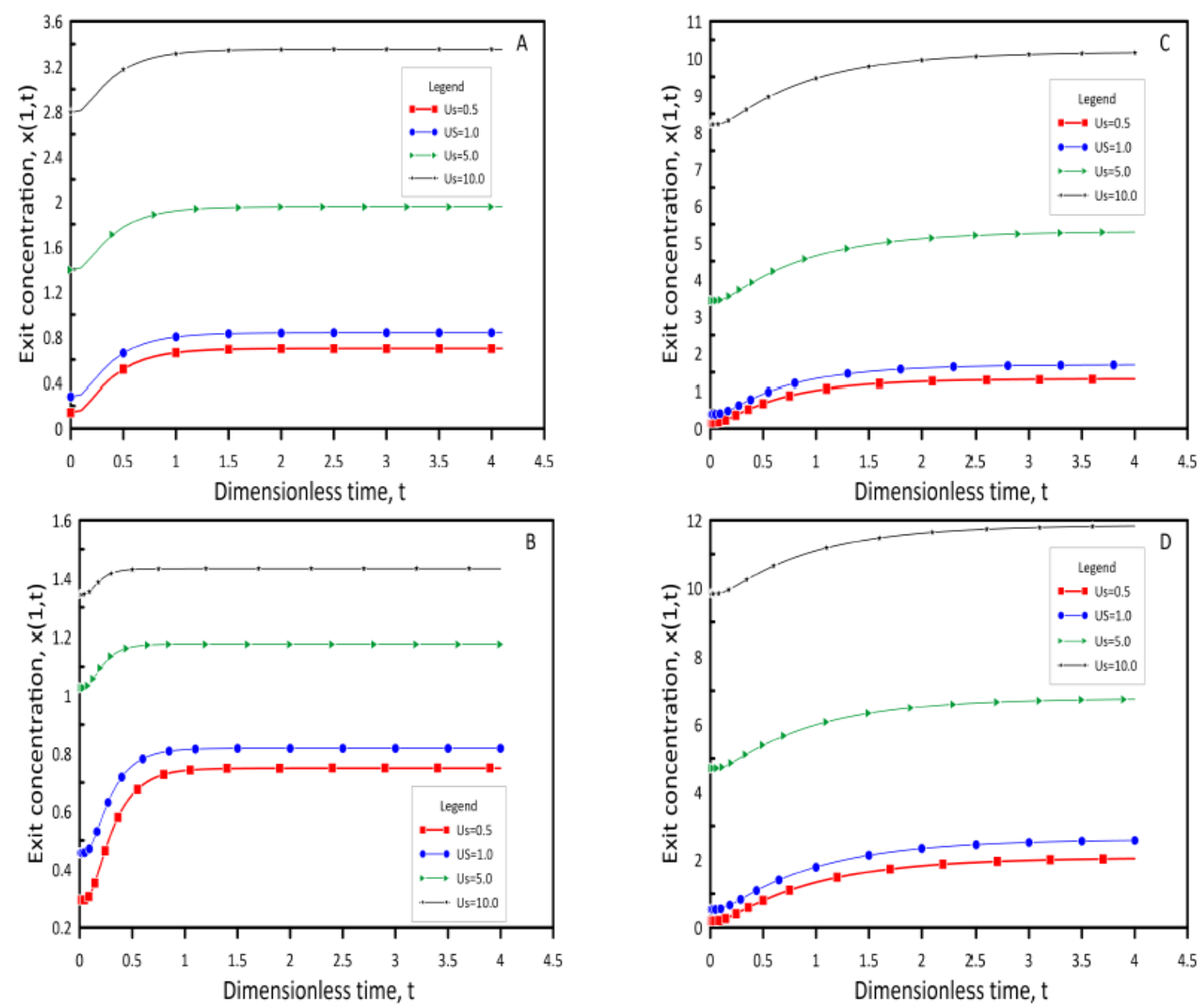

Figure 3: Dynamic response of reactor exit concentration to a step change of +2 units in control input (u) starting from the steady-state given by $\bar{u}$, for the four types of reaction kinetics. A: First-order; B: Second-order; C: Michaelis-Menten and D: Ethylene hydrogenation kinetics. $(\mathrm{Pem}=1.0, \mathrm{Da}=2.0)$,

$$
\bar{u}=u_{s}
$$

The main significant observation of the effect of Pem on the system eigenvalues is that as Pem is increased, the eigenvalues which are all real at low Pem values become a mixture of real and complex conjugate pair eigenvalues at high Pem values. This is expected since, at low Pem values, the reactor mathematical model tends towards a parabolic partial differential equation which can only have real eigenvalues. On the other hand, at high Pem values, the reactor model tends towards a hyperbolic partial differential equation which will have complex eigenvalues due to the characteristic wave nature. 
Table 1: Effect of steady-state control input, $\bar{u}$ on the eigenvalues of the linear or linearized models for the four types of reaction kinetics. Output at $z=1.0,(\mathrm{Da}=2.0, \mathrm{Pem}=1.0) \lambda_{i}=$ ith. eigenvalue of system matrix.

\begin{tabular}{|c|c|c|c|c|c|c|c|c|c|}
\hline $\bar{u}$ & 0.50 & 1.0 & 5.0 & 10.0 & $\bar{u}$ & 0.50 & 1.0 & 5.0 & 10.0 \\
\hline Serial No. & $\lambda_{i}$ & $\lambda_{i}$ & $\lambda_{i}$ & $\lambda_{i}$ & Serial No. & $\lambda_{i}$ & $\lambda_{i}$ & $\lambda_{i}$ & $\lambda_{i}$ \\
\hline (a) & & & & & (c) & & & & \\
\hline 1 & -407.82 & -407.82 & -407.82 & -407.82 & 1 & -407.17 & -406.73 & -405.91 & -405.84 \\
\hline 2 & -357.79 & -357.79 & -357.79 & -357.79 & 2 & -357.18 & -356.75 & -355.89 & -355.82 \\
\hline 3 & -154.46 & -154.46 & -154.46 & -154.46 & 3 & -153.84 & -153.41 & -152.55 & -152.48 \\
\hline 4 & -93.45 & -93.45 & -93.452 & -93.45 & 4 & -92.83 & -92.40 & -91.54 & -91.47 \\
\hline 5 & -43.70 & -4370 & -43.70 & -43.70 & 5 & -43.08 & -42.65 & -41.79 & -41.72 \\
\hline 6 & -14.02 & -14.02 & -14.02 & -14.02 & 6 & -13.40 & -12.96 & -12.11 & -12.04 \\
\hline 7 & -3.17 & -3.17 & -3.17 & -3.17 & 7 & -2.562 & -2.12 & -1.265 & -1.19 \\
\hline (b) & & & & & (d) & & & & \\
\hline 1 & -407.14 & -408.01 & -412.11 & -415.49 & 1 & -406.60 & -406.00 & -405.78 & -405.81 \\
\hline 2 & -357.07 & -357.87 & -361.34 & -363.84 & 2 & -356.62 & -356.00 & -355.79 & -355.78 \\
\hline 3 & -153.73 & -154.52 & -157.89 & -160.30 & 3 & -153.28 & -152.66 & -152.42 & -152.44 \\
\hline 4 & -92.73 & -93.53 & -98.00 & -99.53 & 4 & -92.27 & -91.654 & -91.41 & -91.43 \\
\hline 5 & -42.983 & -43.78 & -47.29 & -49.89 & 5 & -42.52 & -41.90 & -41.66 & -41.68 \\
\hline 6 & -13.313 & -14.13 & -17.83 & -20.73 & 6 & -12.84 & -12.22 & -11.98 & -12.01 \\
\hline 7 & -2.45 & -3.24 & -6.51 & -8.63 & 7 & -3.00 & -1.376 & -1.133 & -1.16 \\
\hline
\end{tabular}

Note: (a), (b), (c) and (d): first-order, second-order, Michaelis-Menten and ethylene hydrogenation kinetics, respectively

The effect of Pem on the simulated transient response of the exit reactor concentration to a step change of +2 units in the inlet control input (u) are shown in Figure 4 for all the four types of reaction kinetics investigated. It can be observed from these plots that the effect of increasing Pem appears to be to slow the response of the exit concentration as it then takes longer to reach and settle at the final steady-states. It can also be observed that for Pem $=100$, the reactor exit concentration dynamic response to the specified step change in the inlet feed concentration displayed some small oscillations during the initial response phase, which then disappeared. This occurred for all the four reaction kinetics under consideration and is the result of the global orthogonal collocation polynomial attempt to approximate the inherent time delay in the PDE reactor models. This kind of oscillation in system dynamic response of global orthogonal collocation lumped model of distributed parameter systems have been reported in a number of previous studies in the literature e.g. [14, 18, 27], the case being that for relatively high values of Pem, the global orthogonal collocation polynomials are not able to provide good approximations of the inherent time delay, and other methods such as orthogonal collocation on finite elements[12] may be considered as the numerical lumping method. For other lower values of Pem shown, no such oscillation exists in the reactor exit concentration dynamic response, implying that the applicable inherent time delays are small, and the global orthogonal collocation polynomials can adequately approximate these. 
Table 2: Effect of Pem on the eigenvalues of the linear or linearized models for the four types of reaction kinetics. Output at $z=1.0,(\mathrm{Da}=2.0, \bar{u}=1.0)$

\begin{tabular}{|c|c|c|c|c|c|c|c|c|c|}
\hline Pem & 0.50 & 1.0 & 10.0 & 100.0 & Pem & 0.50 & 1.0 & 10.0 & 100.0 \\
\hline $\begin{array}{l}\text { Serial } \\
\text { No. }\end{array}$ & $\lambda_{i}$ & $\lambda_{i}$ & $\lambda_{i}$ & $\lambda_{i}$ & $\begin{array}{l}\text { Serial } \\
\text { No. }\end{array}$ & $\lambda_{i}$ & $\lambda_{i}$ & $\lambda_{i}$ & $\lambda_{i}$ \\
\hline (a) & & & & & (c) & & & & \\
\hline 1 & -808.30 & -407.82 & -48.33 & $\begin{array}{c}-5.03+ \\
11.29 j\end{array}$ & 1 & -807.25 & -406.73 & -46.91 & 2003.7 \\
\hline 2 & -709.05 & -357.79 & -36.19 & $\begin{array}{l}-5.03- \\
11.29 \mathrm{j}\end{array}$ & 2 & -708.02 & $\begin{array}{c}- \\
356.755\end{array}$ & -35.39 & $\begin{array}{c}16.63+ \\
19.22 \mathrm{j}\end{array}$ \\
\hline 3 & -305.40 & -154.46 & $\begin{array}{c}-16.24+ \\
2.26 \mathrm{j}\end{array}$ & $\begin{array}{c}-6.62+6.23 \\
j\end{array}$ & 3 & -304.37 & -153.41 & $\begin{array}{c}-15.09+ \\
2.29 \mathrm{j}\end{array}$ & $\begin{array}{l}16.63- \\
19.22 \mathrm{j}\end{array}$ \\
\hline 4 & -182.51 & -93.45 & $\begin{array}{c}-16.24 \text { - } \\
2.26 \mathrm{j}\end{array}$ & $-6.62-6.23 j$ & 4 & -181.48 & -92.400 & $\begin{array}{l}-15.09- \\
2.29 \mathrm{j}\end{array}$ & $\begin{array}{c}3.77+9.99 \\
j\end{array}$ \\
\hline 5 & -83.07 & -43.70 & -10.02 & -11.07 & 5 & -82.048 & -42.655 & -8.89 & $3.77-9.99 \mathrm{j}$ \\
\hline 6 & -23.81 & -14.02 & -6.77 & $-7.61+2.15 j$ & 6 & -22.782 & -12.96 & -5.649 & $5.78+3.29 j$ \\
\hline 7 & -3.089 & -3.17 & -5.02 & $\begin{array}{c}-7.61-2.15 \\
j\end{array}$ & 7 & -2.06 & -2.12 & -3.879 & $5.78-3.29 j$ \\
\hline (b) & & & & & (d) & & & & \\
\hline 1 & -808.39 & -408.01 & -49.57 & $\begin{array}{l}-4.71+ \\
11.20 \mathrm{j}\end{array}$ & 1 & -806.51 & -406.00 & -46.35 & $\begin{array}{l}-3.19+ \\
11.28 \mathrm{j}\end{array}$ \\
\hline 2 & -709.12 & -357.87 & -35.59 & $\begin{array}{l}-4.71- \\
11.20 \mathrm{j}\end{array}$ & 2 & -707.27 & -356.00 & -34.48 & $\begin{array}{l}-3.19- \\
11.28 \mathrm{j}\end{array}$ \\
\hline 3 & -305.44 & $\begin{array}{c}- \\
154.520\end{array}$ & $\begin{array}{c}-16.46+ \\
2.13 \mathrm{j}\end{array}$ & -13.91 & 3 & -303.62 & -152.66 & $\begin{array}{c}-14.36+ \\
2.28 \mathrm{j}\end{array}$ & $\begin{array}{c}-4.74+6.26 \\
j\end{array}$ \\
\hline 4 & -182.56 & -93.534 & $\begin{array}{c}-16.46- \\
2.13 \mathrm{j}\end{array}$ & $\begin{array}{c}-6.50+5.82 \\
j\end{array}$ & 4 & -180.73 & -91.65 & $\begin{array}{l}-14.36- \\
2.28 \mathrm{j}\end{array}$ & $\begin{array}{c}-4.744- \\
6.26 \mathrm{j}\end{array}$ \\
\hline 5 & -83.12 & -43.78 & -10.27 & $\begin{array}{l}-6.50- \\
5.823 \mathrm{j}\end{array}$ & 5 & -81.29 & -41.90 & -8.16 & -9.058 \\
\hline 6 & -23.88 & -14.13 & -7.01 & $\begin{array}{c}-7.59+1.83 \\
j\end{array}$ & 6 & -22.03 & -12.22 & -4.90 & $\begin{array}{c}-5.755+ \\
2.17 \mathrm{j}\end{array}$ \\
\hline 7 & -3.133 & -3.24 & -5.041 & $\begin{array}{c}-7.59-1.83 \\
j\end{array}$ & 7 & -1.31 & -1.38 & -3.15 & $-5.75-2.17 \mathrm{j}$ \\
\hline
\end{tabular}

Note: (a), (b), (c) and (d): first-order, second-order, Michaelis-Menten and ethylene hydrogenation kinetics, respectively 

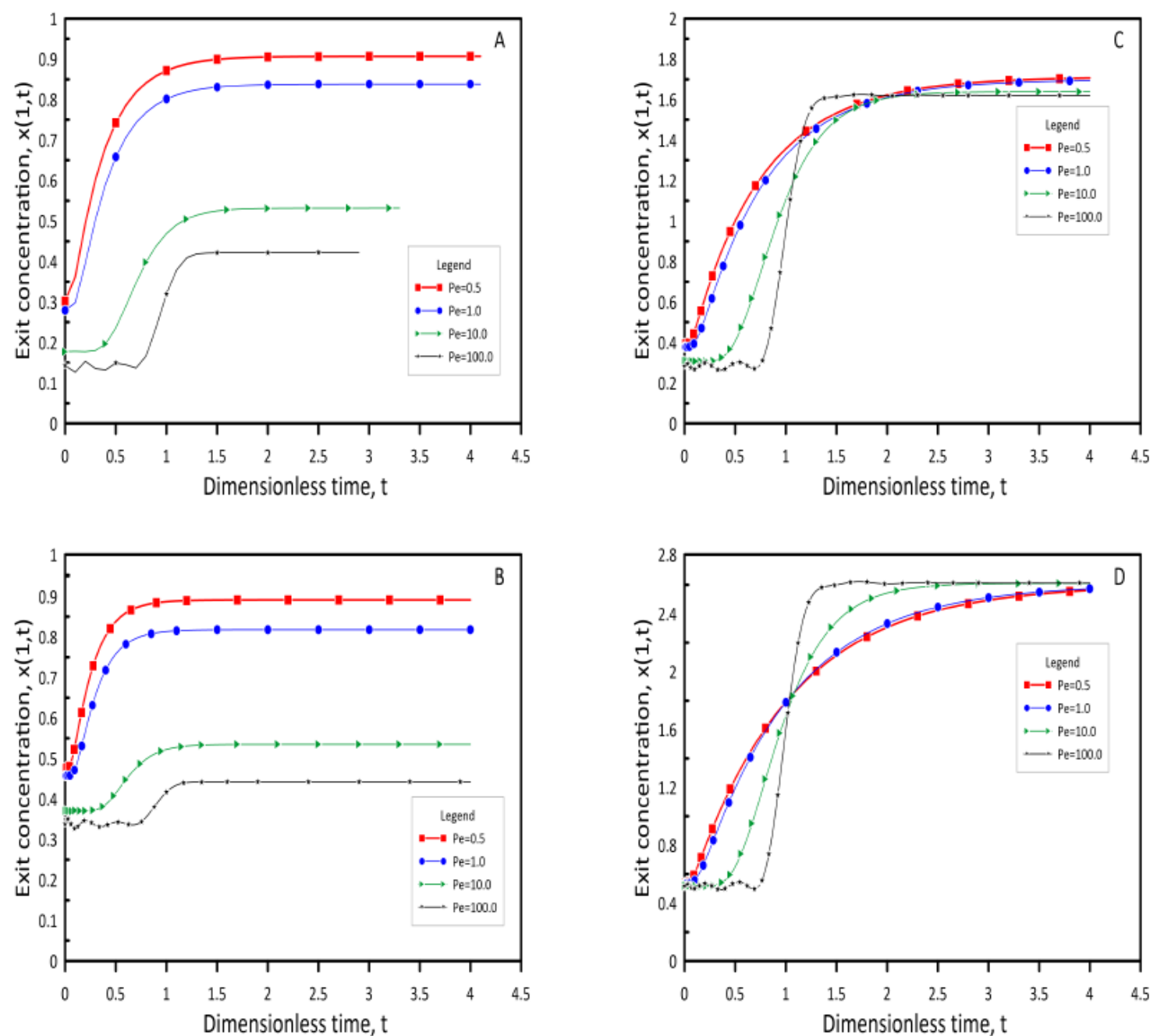

Figure 4: Effect of Pem on the dynamic response of reactor exit concentration to a step change of +2 units in the control input $(u)$. A: First-order; B: Second-order; C: Michaelis-Menten and D: Ethylene hydrogenation kinetics. $(\bar{u}=1.0, \mathrm{Da}=2.0)$

\subsubsection{Effect of Damköhler number, Da}

An examination of the set of eigenvalues (of the linear or linearized models) given in Table 3 shows they are all negative and real. As with the other two cases, this also implies that the reactor is open-loop stable for the four types of reaction kinetics operating at the steady-states defined by the nominal $\bar{u}$, Pem and range of Da considered. Similar to the effect $\bar{u}$, we also observe that the system dynamic response will be dominated by the last three or four eigenvalues with the largest magnitudes. This trend is maintained as $\mathrm{Da}$ is increased from the low to the high value investigated. It can be observed that increasing the value of $\mathrm{Da}$ appears to decrease the dominant time constant of the systems. This trend is exhibited by the transient response simulation of the reactor exit concentration to a step change of +2 units in the reactor inlet concentration shown in Figure 5. It can be observed from these plots that the effect of increasing Da appears to be to increase the response of the exit concentration as it takes a shorter period to reach and settle at the final steady-states. 
Table 3: Effect of Da on the eigenvalues of the linear or linearized models for the four types of reaction kinetics. Output at $z=1.0,(\mathrm{Pem}=1.0, \bar{u}=1.0)$

\begin{tabular}{|c|c|c|c|c|c|c|c|c|c|}
\hline Da & 0.50 & 1.0 & 5.0 & 10.0 & Da & 0.50 & 1.0 & 5.0 & 10.0 \\
\hline $\begin{array}{c}\text { Serial } \\
\text { No. }\end{array}$ & $\lambda_{i}$ & $\lambda_{i}$ & $\lambda_{i}$ & $\lambda_{i}$ & $\begin{array}{c}\text { Serial } \\
\text { No. }\end{array}$ & $\lambda_{i}$ & $\lambda_{i}$ & $\lambda_{i}$ & $\lambda_{i}$ \\
\hline (a) & & & & & (c) & & & & \\
\hline 1 & -406.32 & -406.82 & -410.82 & -415.82 & 1 & -405.97 & -406.18 & -409.04 & -413.51 \\
\hline 2 & -356.29 & -356.79 & -360.79 & -365.79 & 2 & -355.95 & -356.16 & -359.22 & -363.91 \\
\hline 3 & -152.96 & -153.46 & -157.46 & -162.46 & 3 & -152.61 & -152.82 & -155.90 & -160.72 \\
\hline 4 & -91.95 & -92.46 & -96.45 & -101.45 & 4 & -91.60 & -91.81 & -94.86 & -99.61 \\
\hline 5 & -42.20 & -42.70 & -46.70 & -51.70 & 5 & -41.85 & -42.06 & -45.110 & -49.86 \\
\hline 6 & -12.52 & -13.02 & -17.02 & -22.02 & 6 & -12.17 & -12.39 & -15.40 & -20.12 \\
\hline 7 & -1.67 & -2.17 & -6.17 & -11.17 & 7 & -1.33 & -1.54 & -4.58 & -9.31 \\
\hline (b) & & & & & (d) & & & & \\
\hline 1 & -406.57 & -407.14 & -409.87 & -412.11 & 1 & -405.83 & -405.85 & -407.46 & -411.43 \\
\hline 2 & -356.55 & -357.07 & -359.51 & -361.34 & 2 & -355.80 & -355.83 & -357.69 & -362.050 \\
\hline 3 & -153.21 & -153.73 & -156.12 & -157.89 & 3 & -152.46 & -152.49 & -154.31 & -158.85 \\
\hline 4 & -92.20 & -92.73 & -95.16 & -97.00 & 4 & -91.45 & -91.48 & -93.28 & -97.70 \\
\hline 5 & -42.45 & -42.98 & -45.43 & -47.29 & 5 & -41.71 & -41.74 & -43.53 & -47.96 \\
\hline 6 & -12.78 & -13.31 & -15.84 & -17.83 & 6 & -12.03 & -12.06 & -13.84 & -18.29 \\
\hline 7 & -1.92 & -2.45 & -4.82 & -6.51 & 7 & -1.18 & -1.21 & -2.99 & -7.26 \\
\hline
\end{tabular}

Note: (a), (b), (c) and (d): first-order, second-order, Michaelis-Menten and ethylene hydrogenation kinetics, respectively

\section{Conclusion}

The modelling and dynamic simulation of a class of one dimensional, axial dispersion isothermal tubular reactors with PL and LHHW-type kinetics were carried out. Global orthogonal collocation was employed to lump the partial differential equations (PDEs) modelling the reactors with both the PL and LHHW-type kinetics. The resulting lumped parameter models were then used for the dynamic simulations. The results showed that for the nominal design/operating parameters considered, using only 3 or 4 collocation points adequately simulated the dynamic response of the systems. However, simulations over a range of the design/operating parameters required between 5 and 7 collocations points for better results, especially as the Peclet number for mass transfer (Pem) is increased from the nominal value to 100. Examination of the internal dynamics of the system showed that the Peclet number for mass transfer (Pem) has a significant effect on this as exhibited by the types of eigenvalues (real or complex) and the nature of the exit concentration dynamic response. This was not observed to be the case for variations of the inlet feed concentration and the Damkholer number. In comparison with the large order lumped models that result using the finite difference method reported in the literature, the relatively small order of the lumped orthogonal collocation dynamic 
models obtained in this work are useful for dynamic resilience analysis studies and control system analysis/design.
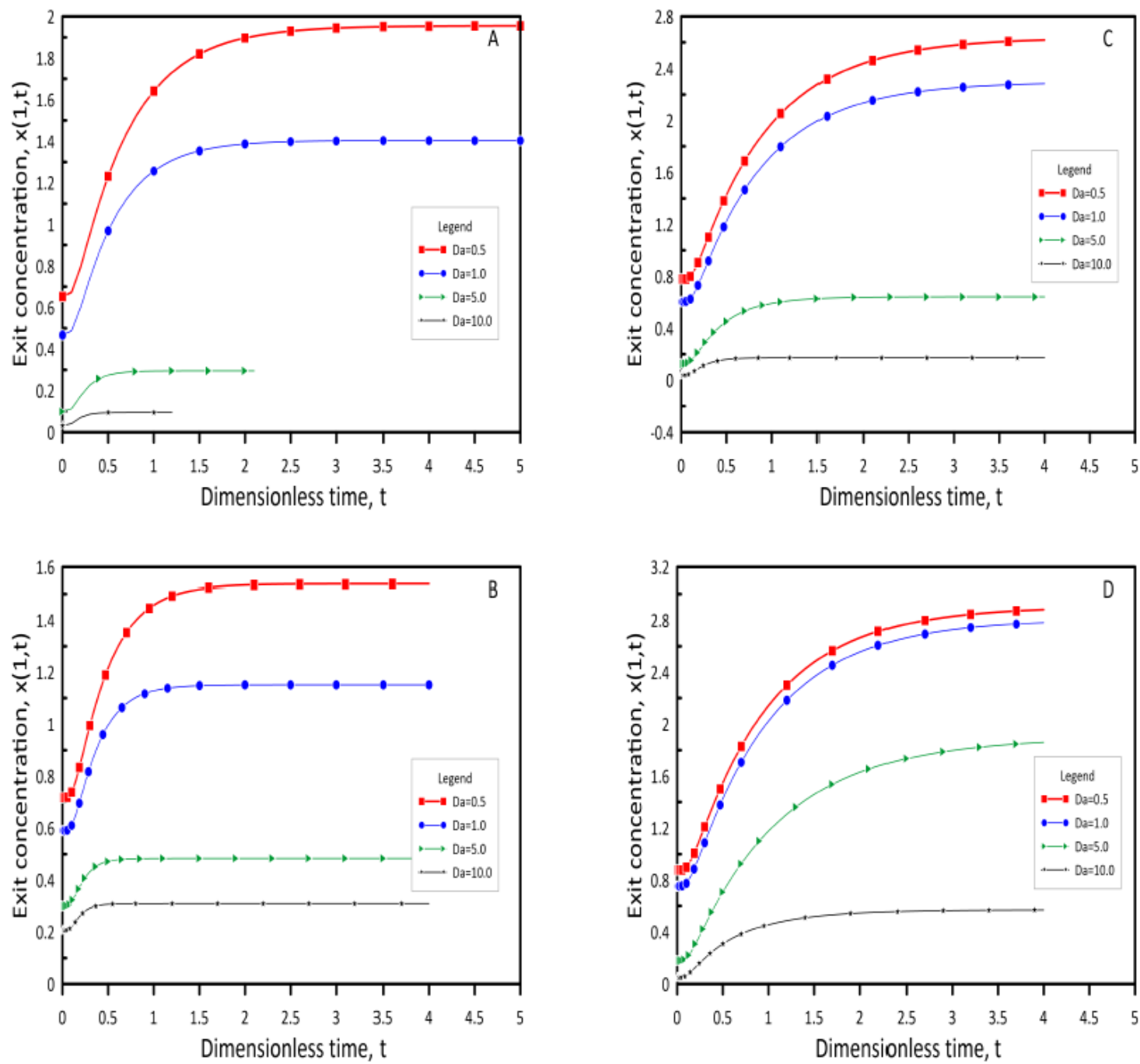

Figure 5: Effect of Da on the dynamic response of reactor exit concentration to a step change of +2 units in the control input $(u)$. A: First-order; B: Second-order; C: Michaelis-Menten and D: Ethylene hydrogenation kinetics. $(\bar{u}=1.0$, Pem $=1.0)$

\section{References}

[1] Pereira, Carmo J. \& Leib, T.M. (2019). Section 19. Reactors. In: Green, Don W., Southard, M.Z., editor. Perry's Chemical Engineers' Handbook; 9th. ed. New York, USA: McGraw-Hill.

[2] Levenspiel, O. (1999). Chemical reaction engineering. 3rd. ed. Wiley. ISBN 9780471254249.

[3] Davis, M.E. \& Davis, R.J.; (2003). Fundamentals of chemical reaction engineering, McGraw-Hill. ISBN 0486488551.

[4] Fogler, H.S. (2016). Elements of chemical reaction engineering. 5th ed. Prentice Hall. ISBN 0133887510. 
[5] Coker, A. (2001). Modeling of chemical kinetics and reactor design. Gulf Professional Pub. ISBN 9780884154815.

[6] Bahadori, A. (2012). Prediction of axial dispersion in plug-flow reactors using a simple method. Journal of Dispersion Science and Technology, 33(2), 200-205.doi:10.1080/01932691.2011.561159.

[7] Ray, W.H. (1981). Advanced process control, McGraw-Hill Companies.

[8] Butt, J.B. (2000). Reaction kinetics and reactor design, Marcel Dekker. ISBN 9780824777227.

[9] Zhou, W., Hamroun, B., Gorrec, Y.L. \& Couenne, F. (2015). Dissipative boundary control systems with application to an isothermal tubular reactor. IFAC-PapersOnLine, 28(13):150-153. doi:10.1016/j.ifacol.2015.10.230.

[10] Li, H.X. \& Qi, C. (2010). Modeling of distributed parameter systems for applications - A synthesized review from time-space separation. Journal of Process Control, 20(8), 891-901. doi:10.1016/j.jprocont.2010.06.016.

[11] Finlayson, B.A. (1972). The method of weighted residuals and variational principles: with application in fluid mechanics, heat and mass transfer, Academic Press. ISBN 9780080955964.

[12] Finlayson, B.A. (2003). Nonlinear analysis in chemical engineering, Ravenna Park Pub. ISBN 096317651X.

[13] Villadsen, J., Michelsen, M.L. (1978). Solution of differential equation models by polynomial approximation. Prentice-Hall.

[14] Dochain, D., Babary, J.P., Tali-Maamar, N. (1992). Modelling and adaptive control of nonlinear distributed parameter bioreactors via orthogonal collocation. Automatica, 28(5), 873-883. doi:10.1016/00051098(92)90141-2.

[15] G'omez, J.C., Viassolo, D.E. \& Junco, S. (1999). Adaptive control of nonlinear distributed parameter systems. International Journal of Modelling and Simulation, 19(2), 194-204. doi:10.1080/02286203.1999.11759971.

[16] Li, M. (2008). Dynamics of axially dispersed reactors. Industrial \& Engineering Chemistry Research, 47(14), 4797-4806. doi:10.1021/ie800083e.

[17] Alopaeus, V., Laavi, H. \& Aittamaa, J. (2008). A dynamic model for plug flow reactor state profiles. Computers and Chemical Engineering, 32(7), 1494-1506. doi:10.1016/j.compchemeng.2007.06.025.

[18] Petre, E. \& Selisteanu, D. (2011). Model approximation and simulations of a class of nonlinear propagation bioprocesses. In: Numerical Analysis - Theory and Application. InTech, 648. doi:10.5772/24131.

[19] Rachidi, S., Karama, A. \& Channa, R. (2014). Modelling of a nonlinear distributed parameter bioreactor via Orthogonal Collocation method. In: International Conference on Multimedia Computing and Systems Proceedings. IEEE Computer Society, 1046- 1050. doi:10.1109/ICMCS.2014.6911140.

[20] Giwa, A. \& Giwa, S.O. (2013). Application of Crank-Nicolson finite-difference method to the solution of the dynamic model of a reactor. International Journal of Advanced Scientific and Technical Research, 6(3), 613-623.

[21] Williams, A.O.F. \& Adeniyi, V.O. (2018). Design of modified IMC-based PID controllers for isothermal tubular reactors with axial mass dispersion and first-order reaction. NSChE Journal, 33(2), 54-54. URL: https://journal.nsche.org.ng/index.php/NSChE/article/view/39.

[22] Williams, A. \& Adeniyi, V. (1996). A new method for the design of PID-Type controllers. IFAC Proceedings Volumes, 29(1), 6125-6130. doi:10.1016/s1474-6670(17)58663-2.

[23] Conesa, J.A. (2020). Chemical reactors design: Mathematical modeling and applications, Wiley-VCH. ISBN 9783527823383.

[24] Elhajaji, A., Barje, N., Serghini, A., Hilal, K. \& Mermri, E.B. (2017). A spline collocation method for integrating a class of chemical reactor equations. International Journal of Nonlinear Analysis and Applications, 8(1), 69-80. doi:10.22075/IJNAA.2017.1653.1436.

[25] Barjes, N., Hajaji, A.E., Serghini, A., Hilal, K. \& Mermri, E.B. (2020). A cubic spline collocation method for integrating a class of chemical reactor equations. Investigacion Operacional, 41(1), 54-66. 
[26] Dochain, D., Tali-Maamar, N. \& Babary, J.P. (1997). On modelling, monitoring and control of fixed bed bioreactors. Computers and Chemical Engineering, 21(11), 1255-1266. doi:10.1016/S00981354(96)00370-5.

[27] Cho, Y.S. \& Joseph, B. (1983). Reduced-order steady-state and dynamic models for separation processes. Part I. Development of the model reduction procedure. AIChE Journal, 29(2), 261- 269. doi:10.1002/aic.690290213.

[28] Danckwerts, P. (1953). Continuous flow systems: Distribution of residence times. Chemical Engineering Science, 2(1), 1-13. doi:10.1016/0009-2509(53)80001-1.

[29] Sheel, J.G.P. \& Crowe, C.M. (1969). Simulation and optimization of an existing ethylbenzene dehydrogenation reactor. The Canadian Journal of Chemical Engineering, 47(2), $183-187$. doi:10.1002/cjce.5450470215.

[30] Rase, H.F. (1990). Fixed-Bed Reactor Design and Diagnostics: Gas-Phase Reactions, Elsevier Science. ISBN 9781483162393.

[31] Elnashaie, S.S.E.H. \& Elshishini, S.S. (1993). Modelling, simulation, and optimization of industrial fixed bed catalytic reactors, Gordon and Breach Science Publishers. ISBN 2881248837.

[32] Iordanidis, A. A. (2002). Mathematical modeling of catalytic fixed bed reactors (pp. 98-112). Enschede, The Netherlands: Twente University Press. ISBN 9036517524.

[33] Elnashaie, S.S.E.H. \& Garhyan, P. (2003). Conservation equations and modeling of chemical and biochemical processes (Google eBook, Marcel Dekker. ISBN 0824709578.

[34] Li, Y., Rangaiah, G.P. \& Ray, A.K. (2003). Optimization of styrene reactor design for two objectives using a genetic algorithm. International Journal of Chemical Reactor Engineering, 1(1). doi:10.2202/15426580.1013.

[35] Yee, A.K., Ray, A.K. \& Rangaiah, G. (2003). Multiobjective optimization of an industrial styrene reactor. Computers \& Chemical Engineering, 27(1), 111-130. doi:10.1016/S0098-1354(02)00163-1.

[36] Babu, B., Chakole, P.G. \& Syed Mubeen, J. (2005). Multiobjective differential evolution (MODE) for optimization of adiabatic styrene reactor. Chemical Engineering Science, 60(17), 4822-4837. doi:10.1016/J.CES.2005.02.073.

[37] Froment, G.F., De Wilde, J. \& Bischoff, K.B. (2011). Chemical Reactor Analysis and Design. Wiley. ISBN 9780470565414.

[38] Srinivasan, A., Depcik, C. (2013).One-dimensional pseudo-homogeneous packed-bed reactor modeling: I. Chemical species equation and effective diffusivity. Chemical Engineering and Technology, 36(1), $22-32$. doi:10.1002/ceat.201200458.

[39] Jakobsen, H.A.; (2014). Chemical Reactor Modeling: Multiphase Reactive Flows: Second Edition, Vol. 9783319050. 2nd ed., Springer International Publishing. doi:10.1007/978-3-319-05092-8.

[40] Chabot, G., Guilet, R., Cognet, P., Gourdon, C. (2015). A mathematical modeling of catalytic milli-fixed bed reactor for Fischer-Tropsch synthesis: Influence of tube diameter on Fischer Tropsch selectivity and thermal behavior. Chemical Engineering Science, 127, 72-83. doi:10.1016/j.ces.2015.01.015.

[41] Froment, G.F. (1974). Fixed bed catalytic reactors. technological and fundamental design aspects. Chemie Ingenieur Technik, 46(9), 374-386. doi:10.1002/cite.330460903.

[42] Luyben, W.L.; 2007. Chemical Reactor Design and Control. John Wiley \& Sons.

[43] Carberry, J.J.; (1976). Chemical and Catalytic Reaction Engineering. New York: McGraw-Hill. ISBN 0486417360.

[44] Panchenkov, G.M., Lebedev, V.B.; (1976). Chemical Kinetics and Catalysis. Translated from Russian.

[45] Matsuura, T., Kato, M. (1967). Concentration stability of the isothermal reactor. Chemical Engineering Science, 22(2), 171-183. doi:10.1016/0009-2509(67)80009-5.

[46] O’Neill, S., Lilly, M., Rowe, P. (1971). Multiple steady states in continuous flow stirred tank enzyme reactors. Chemical Engineering Science, 26(1), 173-175. doi:10.1016/0009-2509(71)86089-X.

[47] Young, L.C. (2019). Orthogonal collocation revisited. Computer Methods in Applied Mechanics and Engineering, 345, 1033-1076. doi:10.1016/J.CMA.2018.10.019. 
[48] Martens, H.R. (1969). A comparative study of digital integration methods. SIMULATION, 12(2), 87-94. doi:10.1177/003754976901200207.

[49] Moler, C., Van Loan, C. (1978). Nineteen dubious ways to compute the exponential of a matrix. SIAM Review, 20(4), 801-836. doi:10.1137/1020098.

[50] Director, S.W., Rohrer, R. (1972). Introduction to Systems Theory. New York, USA: McGraw-Hill.

[51] Williams, A.O.F., Adeniyi, V.O. (2001). Development of some Fortran 77 programs for linear system analysis. NSE Technical Transactions, 36(1), 68-80.

[52] Gerald, C.F. (1978). Applied Numerical Analysis. 2nd. ed.; Reading, MA, Addisson-Wesley.

[53] Lapidus, L.; (1962). Digital Computation for Chemical Engineers. McGraw-Hill.

[54] Georgakis, C., Aris, R., Amundson, N.R. (1977). Studies in the control of tubular reactors-I general considerations. Chemical Engineering Science, 32(11), 1359-1369. doi:10.1016/00092509(77)85032-X. 